$\operatorname{MITP} / 13-017$

\title{
Lattice QCD and the two-photon decay of the neutral pion
}

\author{
Harvey B. Meyer \\ PRISMA Cluster of Excellence, Institut für Kernphysik and Helmholtz Institute Mainz, \\ Johannes Gutenberg-Universität Mainz, D-55099 Mainz
}

\begin{abstract}
Two-photon decays probe the structure of mesons and represent an important contribution to hadronic light-by-light scattering. For the neutral pion, the decay amplitude tests the effects of the chiral anomaly; for a heavy quarkonium state, it measures the magnitude of its wavefunction at the origin. We rederive the expression of the decay amplitude in terms of a Euclidean correlation function starting from the theory defined on the torus. The derivation shows that for timelike photons the approach to the infinite-volume decay amplitude is exponential in the periodic box size.
\end{abstract}

\section{Introduction}

Photon-hadron interactions constitute a venerable subject that continues to be important in 21st century particle physics. Historically, the inner structure of the proton was investigated in elastic scattering experiments of electromagnetic probes [1]. Roughly a decade later, the study of deeply inelastic processes [2,3] led to the development of the parton model, thereby making a decisive contribution to the advent of QCD as the theory of the strong interactions. At the same time, the decay $\pi_{0} \rightarrow \gamma \gamma$ led to the realization that the flavor-singlet chiral symmetry of the classical field theory is broken by quantum effects [4,5]. In this article we rederive the expression for the hadronic matrix element that determines the two-photon width of the neutral pion, starting from QCD defined on a finite torus. This is the context in which almost all lattice QCD calculations are performed. An important conclusion of this analysis is that the finite-size 
effects on the type of hadronic matrix elements that several lattice QCD collaborations are computing [6] 8] are exponentially suppressed.

We assume that QCD has exact isospin symmetry prior to coupling hadrons to photons. We consider a neutral, pseudoscalar meson of mass $M$ which is stable in QCD and which we refer to as the 'pion', although we need not assume that it is a pseudo-Goldstone boson. The basic idea is to think of the square module of the decay amplitude as the pion pole contribution to the light-by-light scattering amplitude. We will be led to consider a center-of-mass energy off by order $e^{2}$ from the pion pole ( $e$ is the electromagnetic coupling constant). At this energy, the $\gamma \gamma$ cross-section is of order $e^{2}$ rather than $e^{4}$. For the sake of the argument, we will consider massive vector bosons $v$ with a finite mass $M_{v} \leq M / 2$ and denote the corresponding field theory by mQED. The QCD part of the $\pi_{0}$ decay amplitude into two timelike photons is reinterpreted as the QCD part of the $\pi_{0}$ decay amplitude into two massive, on-shell vector bosons. We can then use the general formalism developed by Lüscher [9,10] to establish a correspondence between the (discrete) spectrum of $\mathrm{mQED}+\mathrm{QCD}$ on the torus and the $v v$ scattering phase. The $\pi_{0}-v v$ interaction leads to a splitting of the $\pi_{0}$ mass and a two-vectorboson energy level, given by matrix elements of the finite-volume Hamiltonian. The relevant matrix element can then be rewritten in terms of a time-ordered product of vector currents in the finite-volume theory. Since the relation between the finite-volume spectrum and the scattering amplitude holds up to exponentially suppressed corrections, we find that this conclusion also holds for the module of the $\pi_{0} \rightarrow \gamma^{*} \gamma^{*}$ amplitude.

Once the finite-volume corrections have been treated in this way for timelike photons, it is a seemingly innocuous step to extend the conclusion about the finite-size effects to the case of real (lightlike) photons. Rather than giving a formal proof, we check that the relation between the finite-volume matrix element and the infinite-volume matrix element holds even for massless photons in two simples field theories.

One might at first think that, photons being massless, the finite-volume effects will only be suppressed by a power of the linear extent of the torus. However, at leading nontrivial order in the electromagnetic coupling, the photons are merely external particles in the reaction of interest. Their interaction is of purely hadronic origin, and has a range of order the inverse pion Compton wavelength. Inelastic channels are kinematically allowed; but they are suppressed by the smallness of the electromagnetic coupling. We note that the partial wave expansion which underlies Lüscher's formalism is also valid for relativistic and even massless particles (see for instance [11, section 3.7).

Our treatment of the problem bears a strong similarity with the formulation of the $K \rightarrow \pi \pi$ decay on the torus by Lellouch and Lüscher [12]. In some ways, the $\pi_{0} \rightarrow \gamma \gamma$ amplitude is simpler: there are no final-state interactions at order $e^{2}$. In other ways, it is more complicated: the decay amplitude is of second order in the photon-hadron interaction Hamiltonian. The latter induces a shift in the pion mass which is also formally of order $e^{2}$, and which is different in finite and infinite-volume for massless photons (power-law corrections are expected). A second minor complication arises due to the two possible helicities of the final-state photons (either both positive or both 
negative), since there are in principle two open elastic channels. However, due to parity conservation, the two channels are related by a phase difference and one can reduce the problem to a single-channel problem.

The structure of this paper is as follows. The master equation (2.20) relating the finite-volume matrix element to an infinite-volume matrix element is derived for timelike photons in section 2, where it is also brought into a form suited for lattice QCD calculations. In section 3, the pion decay amplitude is calculated in two simple field theories coupled to massless photons, one being an effective description for light quarks and the other for heavy quarks. In section 4, the corresponding finite-volume matrix elements are calculated directly, thus providing a check of the master equation. Unless otherwise stated, we use the notation and conventions of Peskin and Schroeder [13], in particular the metric convention $g_{\mu \nu}=\operatorname{diag}(1,-1,-1,-1)$.

\section{General considerations}

We introduce the amplitude for the decay $\pi_{0} \rightarrow \gamma^{*} \gamma^{*}$. In standard notation (see for instance [14] section 5.1), the amplitude is given by $e^{2}$ times the QCD matrix element

$$
\begin{aligned}
\mathcal{A}_{\mu \nu}(p, q) & =i \int d^{4} x e^{i q \cdot x}\left\langle 0\left|\mathrm{~T}\left\{j_{\mu}(x) j_{\nu}(0)\right\}\right| \pi_{0}(p)\right\rangle_{\mathrm{rel}} \\
& =\epsilon_{\mu \nu \alpha \beta} q^{\alpha} p^{\beta} \mathcal{F}\left(m_{\pi}^{2}, q^{2},(p-q)^{2}\right)
\end{aligned}
$$

where $j^{\mu}$ is the electromagnetic current of hadrons. We have made explicit the use of the 'relativistic' normalization of states,

$$
\operatorname{rel}\left\langle\pi_{0}\left(p^{\prime}\right) \mid \pi_{0}(p)\right\rangle_{\mathrm{rel}}=2 E_{\boldsymbol{p}}(2 \pi)^{3} \delta^{(3)}\left(\boldsymbol{p}-\boldsymbol{p}^{\prime}\right) .
$$

The quantity $\mathcal{F}$ is the off-shell $\pi_{0} \rightarrow \gamma \gamma$ form factor. We will only consider the situation where the pion is on-shell up to higher order corrections and (for simplicity) the case of two photons of same invariant mass,

$$
\mathcal{A} \equiv \epsilon_{\sigma}^{\mu *}(q) \epsilon_{\sigma}^{\nu *}(p-q) \int d^{4} x e^{i q \cdot x}\left\langle 0\left|\mathrm{~T}\left\{j_{\mu}(x) j_{\nu}(0)\right\}\right| \pi_{0}(p)\right\rangle_{\mathrm{rel}}, \quad \sigma=+
$$

with $p^{2}=M^{2}$ and $q^{2}=(p-q)^{2}$. We generically denote by $\epsilon_{\sigma, \mu}(k)$ the polarization vector of the photon. Only the $\sigma= \pm 1$ polarizations will play a role, because the longitudinal polarization $\sigma=0$ does not contribute to the amplitude. The pole contribution to the Lorentz-invariant light-by-light scattering amplitude is then

$$
i \mathcal{M}\left(\gamma_{+} \gamma_{+} \rightarrow \pi_{0} \rightarrow \gamma_{+} \gamma_{+}\right)=-e^{4} \mathcal{A}^{*} \frac{i}{p^{2}-M^{2}} \mathcal{A} .
$$

Except in the propagator, the four-momentum of the pion can be considered to be on-shell. Due to angular momentum conservation, the two photons must have the same helicity. Due to parity conservation, there is a relative minus sign between the 
amplitudes for two positive-helicity photons and the amplitude for two negative-helicity photons in the final state. This information is already encoded in the parametrization (2.2).

We will need the lowest term of the partial-wave expansion of the light-by-light scattering matrix. Due to the two possible helicity states of the two photons in the final state, we are really dealing with a (special case of the) two-channel problem. In the $s$-wave, the partial wave expansion of the amplitude (2.5) reads (see appendix A)

$$
\mathcal{M}=\frac{1}{2} \cdot 16 \pi E \cdot t_{0}, \quad t_{0}=\frac{1}{2 i k}\left(e^{2 i \delta_{0}}-1\right) .
$$

\subsection{Discrete energy eigenstates on the torus}

Our goal is to extract the decay rate of the neutral pion into two photons, $\pi^{0} \rightarrow \gamma \gamma$, from stationary physics on the torus. For the sake of the argument we will however consider massive vector bosons $v$ with a finite mass $M_{v} \leq M / 2$. The QCD part of the $\pi_{0}$ decay amplitude into two timelike photons is reinterpreted as the QCD part of the $\pi_{0}$ decay amplitude into two massive, on-shell vector bosons.

The linear size $L$ of the torus will be assumed to be in the range

$$
\frac{2 \pi}{L} \sim \Lambda \overline{\mathrm{MS}}
$$

In current state-of-the-art lattice QCD calculations, $L$ is typically 3 to $5 \mathrm{fm}$, in which case $2 \pi / L=1240 \mathrm{MeV} / L[\mathrm{fm}]$ is in the ballpark of (2.7). Also, the pion is assumed to fit well into the box, so that the mass difference between the finite-volume theory and the infinite-volume theory can be neglected [15].

We will restrict our attention to the center-of-mass system throughout this paper. We work perturbatively in the electromagnetic coupling $e$. Initially we set $e=0$, so that the vector-boson states and the hadronic states are decoupled. The spectrum of single-vector-boson states is

$$
\left\{\omega_{\boldsymbol{k}} \mid \boldsymbol{k}=\frac{2 \pi}{L} \boldsymbol{n}, \boldsymbol{n} \in \mathbb{Z}^{3}\right\}, \quad \omega_{\boldsymbol{k}}=\sqrt{\boldsymbol{k}^{2}+M_{v}^{2}}
$$

and the spectrum of two-photon states with vanishing total momentum is

$$
\left\{2 \omega_{\boldsymbol{k}} \mid \boldsymbol{k}=\frac{2 \pi}{L} \boldsymbol{n}, \boldsymbol{n} \in \mathbb{Z}^{3}\right\}
$$

The interaction Hamiltonian reads

$$
V=+e \int \mathrm{d} \boldsymbol{x} j^{\mu}(x) A_{\mu}(x) .
$$

The energy levels will be affected by the electromagnetic coupling. Consider first nondegenerate states. At first order in ordinary time-independent perturbation theory, the 
non-degenerate energy levels will change by $\Delta E_{n}^{(1)}=\langle n|V| n\rangle$. We normalize the finitevolume states to have unit norm, $\langle n \mid n\rangle=1$. However, the interaction Hamiltonian changes the vector-boson number by exactly one unit, therefore the matrix elements between states containing the same, definite number of vector bosons vanish. At second order,

$$
\Delta E_{n}^{(2)}=\sum_{m \neq n} \frac{\left|V_{m n}\right|^{2}}{E_{n}^{(0)}-E_{m}^{(0)}} .
$$

If the unperturbed state $|n\rangle$ is a non-degenerate, purely hadronic state, then the states $|m\rangle$ that contribute non-trivially to the sum in Eq. (2.11) are states that contain one vector boson and have an arbitrary hadronic content. For a state $|n\rangle$ containing $N_{n}$ vector bosons, contributions come from states with $N_{n} \pm 1$ vector bosons.

Here we will be interested in the case where the box size is tuned so that the neutral pion mass coincides with the energy of exactly one two-vector-boson state with vanishing total momentum. As is well known, the corrections to the spectrum are found by diagonalizing the perturbing Hamiltonian in the degenerate subspace, which amounts to diagonalizing a finite matrix $V_{n n^{\prime}}$. In this case, the $2 \times 2$ matrix $V_{n n^{\prime}}$ vanishes since there is no pair of states for which the two states differ by a vector boson number of one. To find the leading effect of e.m. interactions on the degenerate $\left|\pi_{0}\right\rangle,|v v\rangle$ states we therefore have to go to second order of degenerate perturbation theory. The recipe is then to diagonalize the matrix ([16], paragraph 39)

$$
W_{n n^{\prime}} \equiv V_{n n^{\prime}}+\sum_{m} \frac{V_{n m} V_{m n^{\prime}}}{E_{n}^{(0)}-E_{m}^{(0)}}
$$

where the sum over $m$ extends over all states not in the degenerate subspace. Since $V_{n n^{\prime}}$ vanishes, the matrix $W_{n n^{\prime}}$ is $\mathrm{O}\left(e^{2}\right)$. We label the neutral pion at rest by ' 1 ' and the two-vector-boson state by ' 2 '. For instance, the matrix element $W_{22}$ corresponds to the transition of one vector boson to a hadronic state and back, while the other one remains as a spectator, a vacuum polarization effect due to the hadrons 1 . The matrix element $W_{11}$ correspond to a mass correction for the pion due to the weakly coupled vector bosons. Its dependence on the volume is exponentially suppressed 2 . The matrix $W_{n n^{\prime}}$ takes the form

$$
W=\left(\begin{array}{cc}
\delta_{L} M & W_{12} \\
W_{12}^{*} & 2 \delta_{L} E_{v}
\end{array}\right)
$$

and the final energy eigenvalues are thus predicted to be, to $\mathrm{O}\left(e^{2}\right)$,

$$
E_{ \pm}=M+\Delta E_{ \pm}=M+\frac{1}{2} \delta_{L} M+\delta_{L} E_{v} \pm \sqrt{\left(\frac{1}{2} \delta_{L} M-\delta_{L} E_{v}\right)^{2}+\left|W_{12}\right|^{2}} .
$$

\footnotetext{
${ }^{1}$ There is a further potential effect, stemming from the fact that the electromagnetic interaction breaks isospin symmetry, which is the mixing of the $\pi_{0}$ with the $\eta$ meson and other, not necessarily isovector states. Since the $\pi_{0}-\eta$ mixing starts at $\mathrm{O}\left(e^{4}\right)$, we can neglect it.

${ }^{2}$ For instance, for a non-relativistic bound state described by a wavefunction $\Psi(\boldsymbol{r})$, it would be $\Delta M=-e^{2} \int d^{3} \boldsymbol{r}|\psi(\boldsymbol{r})|^{2} \frac{e^{-M_{v} r}}{4 \pi r}$ in infinite volume and would only differ by an exponentially small amount on the torus, provided the bound state fits well into the box.
} 


\subsection{Light-by-light scattering near the pole}

Hadronic light-by-light scattering is generically of order $e^{4}$, however for energies a distance $e^{2}$ from the pion pole, the scattering amplitude and the phase shift are of order $e^{2}$. The decay width of the $\pi_{0}$ is of order $e^{4}$ and therefore negligible in the following considerations. However, we must take into account the $\mathrm{O}\left(e^{2}\right)$ shifts in the $\pi_{0}$ mass and the photon mass.

We treat $\delta_{0}$ as a function of the vector boson momentum. For a small phase shift, Eq. (2.5) and (2.6) yield

$$
\delta_{0}\left(k_{ \pm}\right)=-\frac{k e^{4}|\mathcal{A}|^{2}}{16 \pi M^{2}\left(\Delta E_{ \pm}-\delta_{\infty} M\right)} .
$$

\subsection{Relation of the discrete energy levels to the $\pi_{0} \rightarrow v v$ amplitude}

In the finite-volume theory, we work in the basis formed by the state

$$
\left|(v v)_{-}\right\rangle=\frac{1}{2 \sqrt{\nu_{n}}} \sum_{\boldsymbol{k} \in \Omega_{n}} \sum_{\sigma= \pm 1} \sigma a_{\boldsymbol{k} \sigma}^{\dagger} a_{-\boldsymbol{k} \sigma}^{\dagger}|0\rangle
$$

and the state $\left|(v v)_{+}\right\rangle$for which the relative sign between the two helicity terms is positive. The set of momenta appearing in Eq. (2.16) is

$$
\Omega_{n}=\left\{\boldsymbol{k}=2 \pi \boldsymbol{z} / L \mid \boldsymbol{z} \in \mathbb{Z}^{3}, \boldsymbol{z}^{2}=n\right\},
$$

where we restrict ourselves to a value of $n \leq 6$ in order to avoid multiple degeneracies, and $\nu_{n}=\left|\Omega_{n}\right|$ is the cardinality of that set. Only $\left|(v v)_{-}\right\rangle$couples to the $\pi_{0}$, so that we can ignore the state $\left|(v v)_{+}\right\rangle$to the order $e^{2}$ we are working at. The discrete set of momenta $\Gamma$ comprises all the images under the cubic group of one particular allowed momentum on the torus.

Using Lüscher's finite-volume formalism [9, 10, a change in the effective momentum

$k$ defined via the two-vector-boson spectrum, $E=2 \sqrt{M_{v}^{2}+k^{2}}$, from the free-field values (2.9) corresponds to an $s$-wave scattering phase [12,17]

$$
\Delta \delta_{0}(k)=-\left(q \phi^{\prime}(q)+k \frac{\partial \delta_{0}(k)}{\partial k}\right) \frac{\Delta k}{k}, \quad q \equiv \frac{k L}{2 \pi}
$$

with $\phi$ a tabulated kinematic function. Here $\delta_{0}(k)$ vanishes before the vector bosons are coupled to hadrons. Note that the electromagnetic shift in the $\pi_{0}$ pole is contained in $\Delta \delta_{0}$. We use the derivative of $\phi$ at a point where $q=|\boldsymbol{z}|$ for some vector $\boldsymbol{z} \in \mathbb{Z}^{3}$, $q \phi^{\prime}(q)=(2 \pi)^{2} \frac{q^{3}}{\nu_{n}}$. One thus finds

$$
\delta_{0}\left(k_{ \pm}\right)=-\frac{L^{3} k^{2}}{2 \pi \nu_{n}} \Delta k_{ \pm} .
$$


Now the change in the effective momentum of the two vector bosons is related to the spectrum via

$$
\Delta k_{ \pm}=\frac{M}{4 k}\left(\Delta E_{ \pm}-2 \delta_{L} E_{v}\right) .
$$

Combining Eq. (2.15), (2.18) and (2.19), we obtain the master relation

$$
|\mathcal{A}|^{2}=\frac{2 L^{3} M^{3}}{e^{4} \nu_{n}} \cdot\left(\Delta E_{ \pm}-\delta_{\infty} M\right)\left(\Delta E_{ \pm}-2 \delta_{L} E_{v}\right)=\frac{2 L^{3} M^{3}}{e^{4} \nu_{n}} \cdot\left|W_{12}\right|^{2} .
$$

We have used the fact that $\delta_{L} M=\delta_{\infty} M$ up to exponential corrections, and neglected the latter.

\subsection{Explicit expression for the matrix element $W_{12}$}

Using the spectral representation, one verifies that $W_{12}$, defined in Eq. (2.12), is given by

$$
W_{12}=-\frac{1}{2} \int_{-\infty}^{\infty} d t\left\langle(v v)_{-}|\mathrm{T}\{V(t) V(0)\}| B\right\rangle,
$$

as long as there is no contributing intermediate hadronic state with energy $E_{h} \leq M / 2$. The Euclidean time evolution, $V(t)=e^{H t} V(0) e^{-H t}$, is dictated by the full QCD +mQED Hamiltonian $H$. The Wick contractions of the vector bosons can be carried out straightforwardly by noting that in equation (2.12), the intermediate states contributing are direct products of an arbitrary hadronic state with a one-vector-boson state and by using equation (2.16) and the plane-wave expansion of the gauge field. One then reduces $W_{12}$ to a pure QCD matrix element,

$$
W_{12}=-e^{2} \sqrt{\nu_{n}} \epsilon_{+\mu}^{*}(-\boldsymbol{k}) \epsilon_{+\nu}^{*}(\boldsymbol{k}) \int d^{4} x \frac{e^{\omega_{k}\left|x_{0}\right|+i \boldsymbol{k} \cdot \boldsymbol{x}}}{2 \omega_{k}}\left\langle 0\left|\mathrm{~T}\left\{j^{\mu}(x) j^{\nu}(0)\right\}\right| B\right\rangle .
$$

Taking into account the relativistic normalization of states $\left(\sqrt{2 M L^{3}}|B\rangle \rightarrow|B\rangle_{\text {rel. }}\right)$ and inserting (2.22) into (2.20) shows that, possibly up to an overall phase $\varphi$, the $\pi_{0} \rightarrow v v$ amplitude is given by the analytic continuation of Eq. (2.4),

$$
\mathcal{A}=e^{i \varphi} \epsilon_{+\mu}^{*}(-\boldsymbol{k}) \epsilon_{+\nu}^{*}(\boldsymbol{k}) \int d^{4} x e^{\omega_{k}\left|x_{0}\right|+i \boldsymbol{k} \cdot \boldsymbol{x}}\left\langle 0\left|\mathrm{~T}\left\{j^{\mu}(x) j^{\nu}(0)\right\}\right| B\right\rangle_{\mathrm{rel}} \cdot
$$

This expression is suitable for an implementation in lattice QCD [18], see [6] 8] for recent calculations.

\section{The pion decay amplitude in two simple field theories}

In the previous section, relation (2.20) was derived for massive vector bosons and then reduced to a pure QCD matrix element, Eq. (2.23). In the latter equation, the only memory of the dispersion relation of the vector bosons is the relation between $\omega_{k}$ and the spatial momentum $\boldsymbol{k}$. Since the vector bosons merely appear as external legs in 
the amplitude, we expect that the volume corrections affecting the calculation of the amplitude $\mathcal{A}$ in finite volume based on Eq. (2.23) are exponentially small in the box size, even for the case of real photons where $\omega_{k}=|\boldsymbol{k}|$.

In order to check this expectation, in this section we calculate, in two tractable field theories, the amplitude $\mathcal{A}$ for on-shell photons in the final state. We then use relation (2.20) to predict the finite-volume quantity $\left|W_{12}\right|^{2}$. In the next section, the same quantity will be computed directly in finite volume as a check of Eq. (2.20).

\subsection{Theory I: chiral Lagrangian}

In chiral effective theory the decay of a neutral pion of mass $M$ into two photons is driven by the effective Lagrangian (see for instance [19], chapter 22)

$$
\mathcal{L}_{a}=g \pi_{0} \epsilon^{\mu \nu \rho \sigma} F_{\mu \nu} F_{\rho \sigma} .
$$

In the chiral limit the Abelian chiral anomaly [4,5] predicts the value of the coupling [20],

$$
g=\frac{N_{c} e^{2}}{48 \pi^{2} F_{\pi}}
$$

with $F_{\pi} \simeq 184 \mathrm{MeV}$ the pion decay constant and $N_{c}$ the number of colors, however we will not assume any particular value for $g$. In this theory the module of the invariant amplitudes for $\pi_{0}$ decay then amounts to

$$
\left|\mathcal{M}\left(B \rightarrow \gamma_{+} \gamma_{+}\right)\right|^{2}=\left|\mathcal{M}\left(B \rightarrow \gamma_{-} \gamma_{-}\right)\right|^{2}=e^{4}|A|^{2}=16 g^{2} M^{4},
$$

which leads to a full $\gamma \gamma$ width of $\Gamma=M^{3} g^{2} / \pi$.

In order to verify (2.20), we thus have to show by a direct calculation that the module of the finite-volume matrix element $W_{12}$ is given by

$$
\left|W_{12}\right|^{2}=\frac{8 \nu_{n} M g^{2}}{L^{3}}
$$

\subsection{Theory II: a non-relativistic bound state of two massive fermions}

Consider first the amplitude for the fermion-pair annihilation $\bar{f} f \rightarrow \gamma \gamma$. The fermions have a mass $m$. The initial momenta of the fermions are $p$ and $p^{\prime}$ and the final momenta of the photons are $k$ and $k^{\prime}$. There are two diagrams at treelevel, yielding

$$
\mathcal{M}=\frac{e^{2}}{2} \epsilon_{\mu}(k)^{*} \epsilon_{\nu}\left(k^{\prime}\right)^{*} \bar{v}\left(p^{\prime}\right)\left[\gamma^{\nu} \frac{\left(2 p^{\mu}-\not k \gamma^{\mu}\right)}{p \cdot k}+\frac{\gamma^{\mu}\left(2 p^{\nu}-\not k^{\prime} \gamma^{\nu}\right)}{p \cdot k^{\prime}}\right] u(p)
$$

We have used $p^{2}=m^{2}$ and $k^{2}=0$ and the spinor identities

$$
(\not p+m) \gamma^{\nu} u(p)=2 p^{\nu} u(p), \quad \bar{u}(p) \gamma^{\nu}(\not p+m)=\bar{u}(p) 2 p^{\nu} .
$$


Now we choose the center-of-mass frame and consider the limit of non-relativistic fermions. Using standard spinor technology one obtains (for an initial state with one spin-up fermion and one spin-down fermion)

$$
\mathcal{M}=\frac{2 i e^{2}}{m}\left(\boldsymbol{k} \times \boldsymbol{\epsilon}_{\sigma}(\boldsymbol{k})^{*}\right) \cdot \boldsymbol{\epsilon}_{\sigma^{\prime}}(-\boldsymbol{k})^{*} .
$$

For instance, if $\boldsymbol{k}=|\boldsymbol{k}| \boldsymbol{e}_{3}$ and $\boldsymbol{\epsilon}_{\sigma}(\boldsymbol{k})=\frac{1}{\sqrt{2}}(1, i \sigma, 0), \boldsymbol{\epsilon}_{\sigma^{\prime}}(-\boldsymbol{k})=\frac{1}{\sqrt{2}}\left(1,-i \sigma^{\prime}, 0\right)$, then

$$
i\left(\boldsymbol{e}_{3} \times \boldsymbol{\epsilon}_{\sigma}(k)^{*}\right) \cdot \boldsymbol{\epsilon}_{\sigma^{\prime}}\left(k^{\prime}\right)^{*}=-\sigma \delta_{\sigma \sigma^{\prime}} .
$$

Following ([13], chapter 5), we describe a pseudoscalar bound state as 3

$$
|B\rangle=\sqrt{2 M} \int \frac{d^{3} \boldsymbol{p}}{(2 \pi)^{3}} \tilde{\Psi}(\boldsymbol{p}) \frac{1}{\sqrt{2 m}} \frac{1}{\sqrt{2 m}} \frac{1}{\sqrt{2}}\left(|\boldsymbol{p} \uparrow,-\boldsymbol{p} \downarrow\rangle_{\mathrm{rel}}+|\boldsymbol{p} \downarrow,-\boldsymbol{p} \uparrow\rangle_{\mathrm{rel}}\right) .
$$

The amplitude for $B \rightarrow \gamma \gamma$ is then

$$
\begin{aligned}
\mathcal{M}\left(B \rightarrow \gamma_{\sigma} \gamma_{\sigma^{\prime}}\right) & =\sqrt{2 M} \int \frac{d^{3} \boldsymbol{p}}{(2 \pi)^{3}} \frac{\tilde{\Psi}(\boldsymbol{p})}{2 m} \cdot(\mathcal{M}(\boldsymbol{p} \uparrow,-\boldsymbol{p} \downarrow \rightarrow \gamma \gamma)+\mathcal{M}(\boldsymbol{p} \downarrow,-\boldsymbol{p} \uparrow \rightarrow \gamma \gamma)) \\
& =-\sigma \delta_{\sigma \sigma^{\prime}} \frac{2 \sqrt{2} e^{2}}{\sqrt{m}} \Psi(\boldsymbol{x}=0) .
\end{aligned}
$$

As a check, the decay rate of the bound state of mass $M$ into two photons now reads

$$
\begin{aligned}
\Gamma & =\frac{1}{2 M} \int \frac{d^{3} \boldsymbol{k}}{(2 \pi)^{3} 2|\boldsymbol{k}|} \int \frac{d^{3} \boldsymbol{k}^{\prime}}{(2 \pi)^{3} 2\left|\boldsymbol{k}^{\prime}\right|}(2 \pi)^{4} \delta^{(4)}\left(p+p^{\prime}-k-k^{\prime}\right)|\mathcal{M}(B \rightarrow \gamma \gamma)|^{2} \\
& =\frac{e^{4}}{4 \pi m^{2}}|\Psi(\boldsymbol{x}=0)|^{2}
\end{aligned}
$$

In $\Gamma$, we have implicitly included a factor 2 stemming from the sum over final polarization states of the photons, $\sum_{\sigma} \sum_{\sigma^{\prime}} \delta_{\sigma \sigma^{\prime}}=2$, but also a factor $\frac{1}{2 !}$ to remove indistinguishable final-state configurations of the photons. For muonium in the ground state, we have $\Psi(\boldsymbol{x}=0)=\left(\mu^{3 / 2} e^{3}\right) /\left(8 \pi^{2}\right)$, with $\mu=m / 2$ the reduced mass, and the width becomes the well-known result $\Gamma=\alpha^{5} \mathrm{~m} / 2$.

By comparison with Eq. (2.4), the result (3.11) implies in particular

$$
|A|^{2}=\frac{8}{m}|\Psi(\boldsymbol{x}=0)|^{2} .
$$

In order to verify (2.20), we thus have to show by a direct calculation that the module of the finite-volume matrix element $W_{12}$ is given by

$$
\left|W_{12}\right|^{2}=\frac{e^{4} \nu_{n}}{2 L^{3} m^{4}}|\Psi(\boldsymbol{x}=0)|^{2} .
$$

\footnotetext{
${ }^{3}$ The first factor in (3.10) converts the state from a non-relativistic normalization to a relativistic one and we have defined

$$
|\boldsymbol{p} \uparrow,-\boldsymbol{p} \downarrow\rangle=a_{\boldsymbol{p}(+)}^{F \dagger} a_{-\boldsymbol{p}(-)}^{\bar{F} \dagger}|0\rangle, \quad \quad|\boldsymbol{p} \downarrow,-\boldsymbol{p} \uparrow\rangle=a_{\boldsymbol{p ( - )}}^{F \dagger} a_{-\boldsymbol{p}(+)}^{\bar{F} \dagger}|0\rangle,
$$

where $a^{F}\left(a^{\bar{F}}\right)$ is the annihilation operator of the particle (antiparticle). The relative sign between the two terms in (3.10) can be checked by calculating the (non-vanishing) amplitude $\left\langle 0\left|\bar{\psi} \gamma_{5} \psi\right| B\right\rangle$.
} 


\section{Analytic calculation of finite-volume matrix elements}

In order to check the master formula (2.20), we need to determine by a direct calculation the norm of the matrix element $W_{12}$. We work in the Euclidean theory and adopt the corresponding notation. In particular, the Euclidean Dirac matrices are related to the Minkowski space matrices by $\gamma_{0}^{E}=\gamma^{0}$ and $\gamma_{k}^{E}=-i \gamma^{k}, \gamma_{5}^{E}=\gamma_{5}$, but we will not display the superscript 'E' explicitly.

\section{General considerations and the spectral representation}

In order to compute $W_{12}$, we consider the gauge-invariant correlation function

$$
G(t)=\left\langle\mathcal{P}(t) \mathcal{O}_{5}(0)\right\rangle
$$

A gauge-invariant choice of operators would be

$$
\begin{aligned}
\mathcal{P}(t) & =\frac{1}{L^{3 / 2}} \int d^{3} \boldsymbol{x} \epsilon_{\mu \nu \rho \sigma} F_{\mu \nu}(t, \boldsymbol{x}) F_{\rho \sigma}(t, \boldsymbol{x}) \\
\mathcal{O}_{5}(t) & =\frac{1}{L^{3 / 2}} \int d^{3} \boldsymbol{x} \bar{\psi}(t, \boldsymbol{x}) \gamma_{5} \psi(t, \boldsymbol{x}) .
\end{aligned}
$$

In order to simplify the perturbative calculation we will however make a slightly different choice in the next section. We will compute $G(t)$ perturbatively in the electromagnetic interaction at order $e^{2}$. The first non-trivial contribution to $G(t)$ occurs at order $e^{2}$,

$$
\begin{aligned}
G(t) & =G_{0}(t)+e^{2} G_{2}(t)+e^{4} G_{4}(t)+\ldots \\
G_{2}(t) & =\frac{1}{2 !}\left\langle\mathcal{P}(t)\left(\int d^{4} z \mathcal{V}(z)\right)^{2} \mathcal{O}_{5}(0)\right\rangle_{0} \\
\mathcal{V}(x) & =-i e A_{\mu}(x) \bar{\psi}(x) \gamma^{\mu} \psi(x) \\
V(t) & =\int d^{3} \boldsymbol{x} \mathcal{V}(t, \boldsymbol{x})
\end{aligned}
$$

In these equations the (Euclidean) time dependence of the operators is dictated by the non-QED part of the Hamiltonian.

On one hand, the correlation function can be interpreted in terms of the spectral representation. In order to isolate the desired matrix element, we notice that the contribution of interest comes about when the interaction Hamiltonian $\int d^{3} \boldsymbol{x} \mathcal{V}$ is inserted between time 0 and $t$,

$$
\begin{aligned}
G_{2}(t)= & \sum_{n, n^{\prime}}\langle 0|\mathcal{P}(t)| n\rangle \int_{0}^{t} d s e^{-E_{n}(t-s)} \int_{0}^{s} d s^{\prime} \\
& \sum_{l}\langle n|V| l\rangle e^{-E_{l}\left(s-s^{\prime}\right)}\left\langle l|V| n^{\prime}\right\rangle e^{-E_{n^{\prime}} s^{\prime}}\left\langle n^{\prime}\left|\mathcal{O}_{5}(0)\right| 0\right\rangle+\ldots
\end{aligned}
$$

We assume that the torus size has been tuned so that the states $|n\rangle=|\gamma \gamma\rangle$ and $\left|n^{\prime}\right\rangle=|B\rangle$ are degenerate with an energy $E=2|\boldsymbol{k}|$. Carrying out the integrals over $s$ and $s^{\prime}$, their 
contribution to $G_{2}(t)$ will have the following dependence on $t$,

$$
G_{2}(t)=t e^{-E t}(\mathcal{P} \mid \gamma \gamma)\left(B \mid \mathcal{O}_{5}\right)\left(\sum_{l} \frac{\langle\gamma \gamma|V| l\rangle\langle l|V| B\rangle}{E_{l}-E}\right)+\ldots
$$

where for brevity we have defined

$$
(\mathcal{P} \mid \gamma \gamma) \equiv\langle 0|\mathcal{P}(0)| \gamma \gamma\rangle, \quad\left(B \mid \mathcal{O}_{5}\right) \equiv\left\langle B\left|\mathcal{O}_{5}(0)\right| 0\right\rangle .
$$

By comparing to Eq. (2.12), we see that we can extract the matrix element $W_{12}$ from

$$
G_{2}(t)=-W_{12} \cdot(\mathcal{P} \mid \gamma \gamma) \cdot\left(B \mid \mathcal{O}_{5}\right) t e^{-E t}+\ldots
$$

The overlap factors

We use creation and annihilation operators that are unit-normalized, $\left[a_{\underline{p} \underline{\underline{s}}}, a_{\boldsymbol{p}^{\prime} \underline{s}^{\prime}}^{\dagger}\right]_{ \pm}=$ $\delta_{\underline{s s^{\prime}}} \delta_{\boldsymbol{p} \boldsymbol{p}^{\prime}}$ where the plus sign is for bosons and the minus sign for fermions and $\underline{s}$ represents all discrete indices characterizing the particles. The unit-normalized energy eigenstates we are interested in are the following. The two-photon state is defined in Eq. (2.16). For Theory I and II respectively, the 'pseudoscalar meson' states are

$$
\begin{aligned}
|B\rangle & =a_{\boldsymbol{p}=0}^{\dagger}|0\rangle \\
|B\rangle & =\frac{1}{\sqrt{2 L^{3}}} \sum_{\boldsymbol{p}} \tilde{\Psi}(\boldsymbol{p})\left(a_{\boldsymbol{p}(+)}^{F \dagger} a_{-\boldsymbol{p}(-)}^{\bar{F} \dagger}+a_{\boldsymbol{p}(-)}^{F \dagger} a_{-\boldsymbol{p}(+)}^{\bar{F} \dagger}\right)|0\rangle
\end{aligned}
$$

We will use the following spatial Fourier representation and normalization for the wave function,

$$
\Psi(\boldsymbol{x})=\frac{1}{L^{3}} \sum_{\boldsymbol{p}} e^{i \boldsymbol{p} \cdot \boldsymbol{x}} \tilde{\Psi}(\boldsymbol{p}), \quad \int d^{3} \boldsymbol{x}|\Psi(\boldsymbol{x})|^{2}=\frac{1}{L^{3}} \sum_{\boldsymbol{p}}|\tilde{\Psi}(\boldsymbol{p})|^{2}=1 .
$$

Our interpolating operator for the two-photon state will be

$$
\mathcal{P}=A_{i}(-\boldsymbol{k}) A_{j}(\boldsymbol{k}), \quad \text { with } \quad i=1, j=2, \boldsymbol{k}=|\boldsymbol{k}| \boldsymbol{e}_{3} .
$$

Using the plane-wave expansion of the gauge fields,

$$
A_{\mu}(\boldsymbol{x})=\sum_{\boldsymbol{k}, \sigma} \frac{1}{\sqrt{2 \omega_{k} L^{3}}}\left(a_{\boldsymbol{k} \sigma} \epsilon_{\sigma \mu}(\boldsymbol{k}) e^{i \boldsymbol{k} \cdot \boldsymbol{x}}+a_{\boldsymbol{k} \sigma}^{\dagger} \epsilon_{\sigma \mu}^{*}(\boldsymbol{k}) e^{-i \boldsymbol{k} \cdot \boldsymbol{x}}\right),
$$

one finds

$$
\left(\mathcal{P} \mid(\gamma \gamma)_{-}\right)=\frac{i L^{3}}{2 \omega_{k} \sqrt{\nu_{n}}}
$$


for the matrix element. The matter fields have the expansion

$$
\begin{aligned}
\pi_{0}(\boldsymbol{x}) & =\sum_{\boldsymbol{p}} \frac{1}{\sqrt{2 E_{\boldsymbol{p}} L^{3}}}\left(a_{\boldsymbol{p}}^{\pi_{0}} e^{i \boldsymbol{p} \cdot \boldsymbol{x}}+a_{\boldsymbol{p}}^{\pi_{0} \dagger} e^{-i \boldsymbol{p} \cdot \boldsymbol{x}}\right) \\
\psi(\boldsymbol{x}) & =\sum_{\boldsymbol{p}} \frac{1}{\sqrt{2 E_{p} L^{3}}} \sum_{s}\left(a_{\boldsymbol{p} s}^{F} u^{s}(\boldsymbol{p}) e^{i \boldsymbol{p} \cdot \boldsymbol{x}}+a_{\boldsymbol{p} s}^{\bar{F} \dagger} v^{s}(\boldsymbol{p}) e^{-i \boldsymbol{p} \cdot \boldsymbol{x}}\right) \\
\bar{\psi}(\boldsymbol{x}) & =\sum_{\boldsymbol{p}} \frac{1}{\sqrt{2 E_{p} L^{3}}} \sum_{s}\left(a_{\boldsymbol{p} s}^{\bar{F}} \bar{v}^{s}(\boldsymbol{p}) e^{i \boldsymbol{p} \cdot \boldsymbol{x}}+a_{\boldsymbol{p} s}^{F \dagger} \bar{u}^{s}(\boldsymbol{p}) e^{-i \boldsymbol{p} \cdot \boldsymbol{x}}\right)
\end{aligned}
$$

with the normalization $\bar{u}^{r}(\boldsymbol{p}) u^{s}(\boldsymbol{p})=2 m \delta^{r s}$ and $\bar{v}^{r}(\boldsymbol{p}) v^{s}(\boldsymbol{p})=-2 m \delta^{r s}$. In theory I, the interpolating operator is simply the pion field, $\mathcal{O}_{5}=\pi_{0}$, and the overlap is

$$
\left(B \mid \mathcal{O}_{5}\right)=\left(\frac{L^{3}}{2 M}\right)^{1 / 2}
$$

in theory II, the interpolating operator is chosen to be $\mathcal{O}_{5}=-\bar{\psi}(\boldsymbol{p}) \gamma_{5} \psi(-\boldsymbol{p})$ and the overlap is

$$
\left(B \mid \mathcal{O}_{5}\right)=-\sqrt{2 L^{3}} \tilde{\Psi}^{*}(-\boldsymbol{p}) .
$$

Perturbation theory in the time-momentum representation

On the other hand, $G(t)$ can be computed using the standard techniques of Feynman diagrams, using the time-momentum representation of the propagators. The fields are spatially Fourier transformed according to

$$
\begin{aligned}
A_{\mu}(t, \boldsymbol{k}) & =\int d^{3} \boldsymbol{x} A_{\mu}(t, \boldsymbol{x}) e^{-i \boldsymbol{k} \cdot \boldsymbol{x}}, & \pi_{0}(t, \boldsymbol{k}) & =\int d^{3} \boldsymbol{x} \pi_{0}(t, \boldsymbol{x}) e^{-i \boldsymbol{k} \cdot \boldsymbol{x}}, \\
\psi(t, \boldsymbol{k}) & =\int d^{3} \boldsymbol{x} \psi(t, \boldsymbol{x}) e^{-i \boldsymbol{k} \cdot \boldsymbol{x}}, & \bar{\psi}(t, \boldsymbol{k}) & =\int d^{3} \boldsymbol{x} \bar{\psi}(t, \boldsymbol{x}) e^{-i \boldsymbol{k} \cdot \boldsymbol{x}} .
\end{aligned}
$$

In this representation, the photon propagator in Feynman gauge reads

$$
\left\langle A_{\mu}(t, \boldsymbol{k}) A_{\nu}\left(t^{\prime},-\boldsymbol{k}^{\prime}\right)\right\rangle=\delta_{\mu \nu} L^{3} \delta_{\boldsymbol{k} \boldsymbol{k}^{\prime}} \frac{e^{-\omega_{k}\left|t-t^{\prime}\right|}}{2 \omega_{k}},
$$

and the matter fields have the propagators

$$
\begin{aligned}
\left\langle\pi_{0}(t, \boldsymbol{p}) \pi_{0}\left(t^{\prime},-\boldsymbol{k}^{\prime}\right)\right\rangle & =L^{3} \delta_{\boldsymbol{p}^{\prime}} \frac{e^{-E_{\boldsymbol{p}}\left|t-t^{\prime}\right|}}{2 E_{\boldsymbol{p}}} \\
\left\langle\psi(t, \boldsymbol{p}) \bar{\psi}\left(t^{\prime},-\boldsymbol{p}^{\prime}\right)\right\rangle & =L^{3} \delta_{\boldsymbol{p} \boldsymbol{p}^{\prime}}\left(E_{p} \operatorname{sgn}\left(t-t^{\prime}\right) \gamma_{0}-i \boldsymbol{p} \cdot \gamma+m\right) \frac{e^{-E_{p}\left|t-t^{\prime}\right|}}{2 E_{p}} .
\end{aligned}
$$

The fermion-photon vertex is $-i e \gamma_{\mu}$ ( $\mu$ corresponds to the Lorentz index of the photon and $e$ is the charge of the fermion). 


\subsection{Theory I: finite-volume calculation}

At first, we will not make the assumption that the two-photon state is necessarily degenerate with the pseudoscalar matter state. The correlation function at order $e^{2}$ with all fields written in the time-momentum representation reads

$$
\begin{aligned}
G_{2}(t)= & i g \epsilon_{\mu \nu \rho \sigma}\left\langle A_{1}(t,-\boldsymbol{k}) A_{2}(t, \boldsymbol{k})\right. \\
& \left.\quad \int d s \frac{1}{L^{3}} \sum_{\boldsymbol{q}} \pi_{0}(s, \boldsymbol{q}) \frac{1}{L^{3}} \sum_{\boldsymbol{\ell}} F_{\mu \nu}(s, \boldsymbol{l}) F_{\rho \sigma}(s,-(\boldsymbol{l}+\boldsymbol{q})) \pi_{0}(0, \boldsymbol{p}=0)\right\rangle .
\end{aligned}
$$

After all Wick contractions have been performed, one arrives at

$$
G_{2}(t)=-\frac{2 g k_{3} L^{3}}{M \omega_{k}} \int_{-\infty}^{\infty} d s \operatorname{sgn}(t-s) e^{-\left(M|s|+2 \omega_{k}|t-s|\right)} .
$$

Now carrying out the integral over $s$ yields

$$
\int_{-\infty}^{\infty} d s \operatorname{sgn}(t-s) e^{-\left(M|s|+2 \omega_{k}|t-s|\right)}=\frac{e^{-2 \omega_{k} t}-e^{-M t}}{2 \omega_{k}+M}+\frac{e^{-M t}-e^{-2 \omega_{k} t}}{2 \omega_{k}-M},
$$

where the first term comes from the region $s<0$ and $s>t$ and the second from $0<s<t$. If we now take the limit $\omega_{k} \rightarrow M / 2$, the first term vanishes and the second becomes $t e^{-M t}$. Thus the correlation function becomes, for $k_{3}=M / 2$,

$$
G_{2}(t)=-\frac{2 g L^{3}}{M} t e^{-M t}
$$

Comparing it with Eq. (4.11) and dividing out the overlap factors (4.17) and (4.21), we indeed arrive at Eq. (3.4), thus confirming the master relation (2.20).

\subsection{Theory II: finite-volume calculation}

The correlation function at order $e^{2}$ with all fields written in the time-momentum representation reads

$$
\begin{aligned}
& G_{2}(t)=-\frac{e^{2}}{2}\left\langle A_{i}(t,-\boldsymbol{k}) A_{j}(t, \boldsymbol{k})\right. \\
& \int_{-\infty}^{\infty} d s \frac{1}{L^{3}} \sum_{\boldsymbol{\ell}^{\prime}} \frac{1}{L^{3}} \sum_{\boldsymbol{\ell}} A_{\mu}\left(s, \boldsymbol{\ell}^{\prime}\right) \int_{-\infty}^{\infty} d s^{\prime} \frac{1}{L^{3}} \sum_{\boldsymbol{q}^{\prime}} \frac{1}{L^{3}} \sum_{\boldsymbol{q}} A_{\nu}\left(s, \boldsymbol{q}^{\prime}\right)\left(\gamma_{5}\right)_{\beta \alpha} \\
& \left.\bar{\psi}(s, \boldsymbol{\ell}) \gamma^{\mu} \psi\left(s,-\left(\boldsymbol{\ell}^{\prime}+\boldsymbol{\ell}\right)\right) \bar{\psi}\left(s^{\prime}, \boldsymbol{q}\right) \gamma^{\nu} \psi\left(s^{\prime},-\left(\boldsymbol{q}^{\prime}+\boldsymbol{q}\right)\right) \psi_{\alpha}(0,-\boldsymbol{p}) \bar{\psi}_{\beta}(0, \boldsymbol{p})\right\rangle
\end{aligned}
$$

The Wick contractions of the gauge fields lead to

$$
\begin{aligned}
& G_{2}(t)=-\frac{e^{2}}{2} \int_{-\infty}^{\infty} d s \int_{-\infty}^{\infty} d s^{\prime} \frac{e^{-\omega_{\boldsymbol{k}}|t-s|}}{2 \omega_{\boldsymbol{k}}} \frac{e^{-\omega_{\boldsymbol{k}}\left|t-s^{\prime}\right|}}{2 \omega_{\boldsymbol{k}}} \frac{1}{L^{3}} \sum_{\boldsymbol{\ell}} \frac{1}{L^{3}} \sum_{\boldsymbol{q}}\left(\gamma_{5}\right)_{\beta \alpha} \\
& \left\langle\bar{\psi}(s, \boldsymbol{\ell}) \gamma^{i} \psi(s,-(\boldsymbol{\ell}+\boldsymbol{k})) \bar{\psi}\left(s^{\prime}, \boldsymbol{q}\right) \gamma^{j} \psi\left(s^{\prime}, \boldsymbol{k}-\boldsymbol{q}\right) \psi_{\alpha}(0,-\boldsymbol{p}) \bar{\psi}_{\beta}(0, \boldsymbol{p})\right\rangle \\
& \quad+(i \leftrightarrow j, \boldsymbol{k} \rightarrow-\boldsymbol{k}) .
\end{aligned}
$$


The fermion propagator joining the two insertions of the vector current is treated as a free propagator,

$$
\begin{gathered}
G_{2}(t)=-e^{2} \int_{-\infty}^{\infty} d s \int_{-\infty}^{\infty} d s^{\prime} \frac{e^{-\omega_{\boldsymbol{k}}|t-s|}}{2 \omega_{\boldsymbol{k}}} \frac{e^{-\omega_{\boldsymbol{k}}\left|t-s^{\prime}\right|}}{2 \omega_{\boldsymbol{k}}} \gamma_{\gamma \delta}^{i} \gamma_{\gamma^{\prime} \delta^{\prime}}^{j}\left(\gamma_{5}\right)_{\beta \alpha} \\
\frac{1}{L^{3}} \sum_{\boldsymbol{q}} \frac{e^{-E_{\boldsymbol{q}+\boldsymbol{k}}\left|s-s^{\prime}\right|}}{2 E_{\boldsymbol{q}+\boldsymbol{k}}}\left(E_{\boldsymbol{q}+\boldsymbol{k}} \operatorname{sgn}\left(s-s^{\prime}\right) \gamma_{0}+i \boldsymbol{\gamma} \cdot(\boldsymbol{q}+\boldsymbol{k})+m\right)_{\delta \gamma^{\prime}} \\
\quad\left\langle\bar{\psi}_{\gamma}(s, \boldsymbol{q}) \psi_{\delta^{\prime}}\left(s^{\prime},-\boldsymbol{q}\right) \psi_{\alpha}(0,-\boldsymbol{p}) \bar{\psi}_{\beta}(0, \boldsymbol{p})\right\rangle \\
+(i \leftrightarrow j, \boldsymbol{k} \rightarrow-\boldsymbol{k}) .
\end{gathered}
$$

We can now use expression (B.6) for the fermion four-point function that takes into account the presence of a bound state in the spectrum (see appendix B) and obtain

$$
\begin{aligned}
& G_{2}(t)=2 e^{2} \tilde{\Psi}(-\boldsymbol{p})^{*}\left(\bar{u}^{(+)}(-\boldsymbol{p}) \gamma_{5} v^{(-)}(\boldsymbol{p})+\bar{u}^{(-)}(-\boldsymbol{p}) \gamma_{5} v^{(+)}(\boldsymbol{p})\right) \\
& \quad \int_{-\infty}^{\infty} d s \int_{-\infty}^{s} d s^{\prime} \frac{e^{-\omega_{\boldsymbol{k}}|t-s|}}{2 \omega_{\boldsymbol{k}}} \frac{e^{-\omega_{\boldsymbol{k}}\left|t-s^{\prime}\right|}}{2 \omega_{\boldsymbol{k}}} \frac{e^{-E_{B} s^{\prime}}}{2 \cdot 2 E_{p}} \sum_{\boldsymbol{q}} \frac{e^{-E_{\boldsymbol{q}+\boldsymbol{k}}\left(s-s^{\prime}\right)}}{2 E_{\boldsymbol{q}+\boldsymbol{k}}} \frac{e^{-E_{\boldsymbol{q}}\left(s-s^{\prime}\right)}}{2 E_{\boldsymbol{q}}} \tilde{\Psi}(-\boldsymbol{q}) \\
& \quad \sum_{r= \pm} \bar{v}^{r}(\boldsymbol{q}) \gamma^{i}\left(E_{\boldsymbol{q}+\boldsymbol{k}} \operatorname{sgn}\left(s-s^{\prime}\right) \gamma_{0}+i \boldsymbol{\gamma} \cdot(\boldsymbol{q}+\boldsymbol{k})+m\right) \gamma^{j} u^{-r}(-\boldsymbol{q}) \\
& \quad+(i \leftrightarrow j, \boldsymbol{k} \rightarrow-\boldsymbol{k})+\ldots
\end{aligned}
$$

The dots stand for terms which do not contribute to the contribution proportional to $t e^{-E t}$ that we are interested in. Now since the wavefunction $\tilde{\Psi}(\boldsymbol{q})$ is concentrated at momenta much less than $m$ in norm, we can approximate all the factors at leading order by evaluating them at $\boldsymbol{q}=0$. After this operation, the first two lines in Eq. (4.36) are an even function of $\boldsymbol{k}$, hence we can evaluate the spinor matrix element where we now set $i=1, j=2$ and $\boldsymbol{k}=\mathrm{e}_{3}$,

$$
\begin{aligned}
& \sum_{r= \pm} \bar{v}^{r}(\mathbf{0}) \gamma^{i}\left(E_{\boldsymbol{k}} \operatorname{sgn}\left(s-s^{\prime}\right) \gamma_{0}+i \boldsymbol{\gamma} \cdot \boldsymbol{k}+m\right) \gamma^{j} u^{-r}(\mathbf{0})+(i \leftrightarrow j, \boldsymbol{k} \rightarrow-\boldsymbol{k}) \\
& \quad=-2 i m \sum_{r= \pm} v^{r}(\mathbf{0})^{\dagger} \gamma_{5} u^{-r}(\mathbf{0})=8 i m^{2} \\
& \bar{u}^{(+)}(\mathbf{0}) \gamma_{5} v^{(-)}(\mathbf{0})+\bar{u}^{(-)}(\mathbf{0}) \gamma_{5} v^{(+)}(\mathbf{0})=-4 m .
\end{aligned}
$$

Now the integrals over $s$ and $s^{\prime}$ can be carried out, yielding (for $i=1$ and $j=2$ )

$$
G_{2}(t)=-i \Psi(\mathbf{0}) \tilde{\Psi}(-\boldsymbol{p})^{*} \frac{e^{2} L^{3} t e^{-2 m t}}{2 m^{3}}+\ldots
$$

We assume that the wavefunction $\Psi$ of the bound state on the torus only differs by a negligible amount from the infinite volume wavefunction. For $L$ sufficiently large this assumption is justified by the general analysis [15]. Comparing with Eq. (4.11) and dividing by the overlap factors (4.22) and (4.17), we arrive at the expected formula (3.14) and have thus confirmed the master relation (2.20). 


\section{Final remarks}

The approach followed here relies on the relation between the discrete two-particle spectrum on the torus and the $S$-matrix of the corresponding infinite-volume theory $[9$, [10. Since this relation is only guaranteed to hold up to exponential corrections in the volume for massive field theories, we were led to consider massive vector bosons. However, in the final QCD matrix element (2.23), the dispersion relation of the vector bosons only appears to play a secondary role. It would therefore be desirable to have a more direct approach to the question of finite-size corrections of the $\pi_{0} \rightarrow \gamma \gamma$ decay amplitude, presumably using some of the mathematical techniques developed in [9, 21, 22 .

In isospin-symmetric $\mathrm{QCD}$, the $\eta$ is perfectly stable, and it would be interesting to study the case $\eta \rightarrow \gamma \gamma$ along the same lines we followed here. One must however take into account that at $\sqrt{s}=M_{\eta}, \gamma \gamma \rightarrow 3 \pi$ is kinematically allowed and of the same order in the fine structure constant as the $\eta$ pole contribution to light-by-light scattering. Unless one makes use of the specific dynamics of pions [23], one is then dealing with a case involving an open inelastic channel. The theoretical framework to study such problems is under construction [24,25].

\section{Acknowledgments}

I thank Stefan Scherer for encouragement and interesting discussions. This work was supported by the Center for Computational Sciences in Mainz.

\section{A Reduction to a one-channel problem}

We consider the elastic scattering of two vector bosons with same helicity. In the final state, the vector bosons can have either both positive, or both negative helicity, and there are thus two open channels. We follow the notation of Sharpe and Hansen [22].

The S-matrix is written as

$$
i(\mathcal{M})_{\mathrm{B}}=K\left((S)_{\mathrm{B}}-1\right), \quad K=\frac{8 \pi E}{k} .
$$

In the basis $B=\left(\left|v_{+} v_{+}\right\rangle,\left|v_{-} v_{-}\right\rangle\right)$, we saw that the scattering amplitude takes the form

$$
i(\mathcal{M})_{\mathrm{B}}=i\left(\begin{array}{cc}
\mathcal{M} & -\mathcal{M} \\
-\mathcal{M} & \mathcal{M}
\end{array}\right)
$$

On the other hand, in order to relate $\mathcal{M}$ to a phase shift, we realize that in the basis $B^{\prime}=\left(\left|(v v)_{+}\right\rangle,\left|(v v)_{-}\right\rangle\right)$, the S-matrix is given by

$$
(S)_{\mathrm{B}^{\prime}}=\operatorname{diag}\left(e^{2 i \delta_{0}}, 0\right) .
$$


Performing the change of basis,

$$
i(\mathcal{M})_{\mathrm{B}}=K\left[\frac{1}{2}\left(\begin{array}{cc}
1 & 1 \\
-1 & 1
\end{array}\right)\left(\begin{array}{cc}
e^{2 i \delta_{0}} & 0 \\
0 & 1
\end{array}\right)\left(\begin{array}{cc}
1 & -1 \\
1 & 1
\end{array}\right)-\left(\begin{array}{ll}
1 & 0 \\
0 & 1
\end{array}\right)\right]
$$

the scattering amplitude in the basis $B$ thus takes the form (A.2) with

$$
\mathcal{M}=K \frac{e^{2 i \delta_{0}}-1}{2 i}
$$

This is precisely the expression given in Eq. (2.6).

\section{B The fermion four-point function}

Using the plane-wave expansion of the quantum fields $\psi$ and $\bar{\psi}$, we can write the twofermion correlation function as

$$
\begin{aligned}
& \left\langle\bar{\psi}_{\gamma}\left(t, \boldsymbol{x}^{\prime}\right) \psi_{\delta^{\prime}}\left(t^{\prime}, \boldsymbol{y}^{\prime}\right) \psi_{\alpha}(0,-\boldsymbol{p}) \bar{\psi}_{\beta}(0, \boldsymbol{p})\right\rangle \\
& \stackrel{t>t^{\prime}>0}{=} \frac{1}{2 E_{p}} \sum_{\boldsymbol{p}^{\prime \prime}, \boldsymbol{p}^{\prime}} \sum_{s, s^{\prime}, s^{\prime \prime}, s^{\prime \prime \prime}} e^{-E_{\boldsymbol{p}^{\prime \prime}}\left(t-t^{\prime}\right)}\left\langle 0\left|a_{\boldsymbol{p}^{\prime \prime} s^{\prime \prime}}^{\bar{F}} a_{\boldsymbol{p}^{\prime} s^{\prime \prime \prime}}^{F} e^{-H t^{\prime}} a_{\boldsymbol{p} s}^{\bar{F}} a_{(-\boldsymbol{p}) s^{\prime}}^{F}\right| 0\right\rangle \\
& \quad e^{i\left(\boldsymbol{p}^{\prime \prime} \cdot \boldsymbol{x}^{\prime}+\boldsymbol{p}^{\prime} \cdot \boldsymbol{y}^{\prime}\right)} \frac{\bar{v}_{\gamma}^{s^{\prime \prime}}\left(\boldsymbol{p}^{\prime \prime}\right) u_{\delta^{\prime}}^{s^{\prime \prime \prime}}\left(\boldsymbol{p}^{\prime}\right) v_{\alpha}^{s}(\boldsymbol{p}) \bar{u}_{\beta}^{s^{\prime}}(-\boldsymbol{p})}{\sqrt{2 E_{p^{\prime \prime}} 2 E_{p^{\prime}}}} .
\end{aligned}
$$

Now we insert a complete set of states in the remaining matrix element, the contribution of a bound state $|B\rangle$ is

$$
\left\langle 0\left|a_{\boldsymbol{p}^{\prime \prime} s^{\prime \prime}}^{\overline{f^{\prime}}} a_{\boldsymbol{p}^{\prime} s^{\prime \prime \prime}}^{F}\right| B\right\rangle e^{-E_{B} t^{\prime}}\left\langle B\left|a_{\boldsymbol{p} s}^{\bar{F}} a_{(-\boldsymbol{p}) s^{\prime}}^{F}\right| 0\right\rangle .
$$

The individual matrix elements are given by

$$
\begin{aligned}
\left\langle 0\left|a_{\boldsymbol{p}^{\prime \prime} s^{\prime \prime}}^{\bar{P}} a_{\boldsymbol{p}^{\prime} s^{\prime \prime \prime}}^{F}\right| B\right\rangle & =\frac{1}{\sqrt{2 L^{3}}} \tilde{\Psi}\left(\boldsymbol{p}^{\prime}\right) \delta_{\boldsymbol{p}^{\prime \prime}\left(-\boldsymbol{p}^{\prime}\right)}\left(\delta_{s^{\prime \prime}(-)} \delta_{s^{\prime \prime \prime}(+)}+\delta_{s^{\prime \prime}(+)} \delta_{s^{\prime \prime \prime}(-)}\right), \\
\left\langle B\left|a_{\boldsymbol{p} s}^{\bar{F}} a_{(-\boldsymbol{p}) s^{\prime}}^{F}\right| 0\right\rangle & =\frac{1}{\sqrt{2 L^{3}}}\left(-\tilde{\Psi}^{*}(-\boldsymbol{p})\right)\left(\delta_{s^{\prime}(+)} \delta_{s(-)}+\delta_{s^{\prime}(-)} \delta_{s(+)}\right) .
\end{aligned}
$$

Finally, we obtain the expression for $t^{\prime} \rightarrow \infty$

$$
\begin{aligned}
& \left\langle\bar{\psi}_{\gamma}\left(t, \boldsymbol{x}^{\prime}\right) \psi_{\delta^{\prime}}\left(t^{\prime}, \boldsymbol{y}^{\prime}\right) \psi_{\alpha}(0,-\boldsymbol{p}) \bar{\psi}_{\beta}(0, \boldsymbol{p})\right\rangle \\
& =\frac{-1}{L^{3}} \tilde{\Psi}(-\boldsymbol{p})^{*} \frac{e^{-E_{B} t^{\prime}}}{2 \cdot 2 E_{p}}\left(v_{\alpha}^{(-)}(\boldsymbol{p}) \bar{u}_{\beta}^{(+)}(-\boldsymbol{p})+v_{\alpha}^{(+)}(\boldsymbol{p}) \bar{u}_{\beta}^{(-)}(-\boldsymbol{p})\right) \\
& \quad \sum_{\boldsymbol{p}^{\prime \prime}} \frac{e^{-E_{p^{\prime \prime}}\left(t-t^{\prime}\right)}}{2 E_{p^{\prime \prime}}} e^{i \boldsymbol{p}^{\prime \prime} \cdot\left(\boldsymbol{x}^{\prime}-\boldsymbol{y}^{\prime}\right)} \tilde{\Psi}\left(-\boldsymbol{p}^{\prime \prime}\right)\left(\bar{v}_{\gamma}^{(-)}\left(\boldsymbol{p}^{\prime \prime}\right) u_{\delta^{\prime}}^{(+)}\left(-\boldsymbol{p}^{\prime \prime}\right)+\bar{v}_{\gamma}^{(+)}\left(\boldsymbol{p}^{\prime \prime}\right) u_{\delta^{\prime}}^{(-)}\left(-\boldsymbol{p}^{\prime \prime}\right)\right) .
\end{aligned}
$$


Equivalently, we can transform all fermion fields to the time-momentum representation and then obtain

$$
\begin{aligned}
& \left\langle\bar{\psi}_{\gamma}(t, \boldsymbol{k}) \psi_{\delta^{\prime}}\left(t^{\prime},-\boldsymbol{q}^{\prime}\right) \psi_{\alpha}(0,-\boldsymbol{p}) \bar{\psi}_{\beta}(0, \boldsymbol{p})\right\rangle \\
& =-L^{3} \delta_{\boldsymbol{k} \boldsymbol{q}^{\prime}} \tilde{\Psi}(-\boldsymbol{p})^{*} \frac{e^{-E_{B} t^{\prime}}}{2 \cdot 2 E_{p}}\left(v_{\alpha}^{(-)}(\boldsymbol{p}) \bar{u}_{\beta}^{(+)}(-\boldsymbol{p})+v_{\alpha}^{(+)}(\boldsymbol{p}) \bar{u}_{\beta}^{(-)}(-\boldsymbol{p})\right) . \\
& \quad \frac{e^{-E_{\boldsymbol{k}}\left(t-t^{\prime}\right)}}{2 E_{\boldsymbol{k}}} \tilde{\Psi}(-\boldsymbol{k})\left(\bar{v}_{\gamma}^{(-)}(\boldsymbol{k}) u_{\delta^{\prime}}^{(+)}(-\boldsymbol{k})+\bar{v}_{\gamma}^{(+)}(\boldsymbol{k}) u_{\delta^{\prime}}^{(-)}(-\boldsymbol{k})\right),
\end{aligned}
$$

\section{References}

[1] R. Hofstadter and R. W. McAllister, Electron scattering from the proton, Phys. Rev. 98 (Apr, 1955) 217-218.

[2] E. D. Bloom, D. H. Coward, H. DeStaebler, J. Drees, G. Miller, L. W. Mo, R. E. Taylor, M. Breidenbach, J. I. Friedman, G. C. Hartmann, and H. W. Kendall, High-energy inelastic $e-p$ scattering at $6^{\circ}$ and $10^{\circ}$, Phys. Rev. Lett. 23 (Oct, 1969) 930-934.

[3] M. Breidenbach, J. I. Friedman, H. W. Kendall, E. D. Bloom, D. H. Coward, H. DeStaebler, J. Drees, L. W. Mo, and R. E. Taylor, Observed behavior of highly inelastic electron-proton scattering, Phys. Rev. Lett. 23 (Oct, 1969) 935-939.

[4] S. L. Adler, Axial vector vertex in spinor electrodynamics, Phys.Rev. 177 (1969) $2426-2438$.

[5] J. Bell and R. Jackiw, A PCAC puzzle: $\pi_{0} \rightarrow \gamma \gamma$ in the sigma model, Nuovo Cim. A60 (1969) 47-61.

[6] X. Feng, S. Aoki, H. Fukaya, S. Hashimoto, T. Kaneko, et. al., Two-photon decay of the neutral pion in lattice QCD, Phys.Rev.Lett. 109 (2012) 182001, arXiv:1206.1375.

[7] H.-W. Lin and S. D. Cohen, Neutral Meson Decays into Two Photons from Lattice QCD, arXiv:1302.0874

[8] X. Feng, S. Aoki, H. Fukaya, S. Hashimoto, T. Kaneko, et. al., The neutral pion decay and the chiral anomaly on the lattice, PoS LATTICE2012 (2012) 180, arXiv:1211.2504.

[9] M. Lüscher, Volume Dependence of the Energy Spectrum in Massive Quantum Field Theories. 2. Scattering States, Commun.Math.Phys. 105 (1986) 153-188.

[10] M. Lüscher, Two particle states on a torus and their relation to the scattering matrix, Nucl.Phys. B354 (1991) 531-578.

[11] S. Weinberg, The Quantum theory of fields. Vol. 1: Foundations, . Cambridge, UK: Univ. Pr. (1995) 609 p.

[12] L. Lellouch and M. Lüscher, Weak transition matrix elements from finite volume correlation functions, Commun.Math.Phys. 219 (2001) 31-44, hep-lat/0003023.

[13] M. E. Peskin and D. V. Schroeder, An Introduction to quantum field theory, . Reading, USA: Addison-Wesley $842 \mathrm{p}$. 
[14] F. Jegerlehner and A. Nyffeler, The Muon g-2, Phys.Rept. 477 (2009) 1-110, arXiv:0902.3360.

[15] M. Lüscher, Volume Dependence of the Energy Spectrum in Massive Quantum Field Theories. 1. Stable Particle States, Commun.Math.Phys. 104 (1986) 177.

[16] L. Landau and E. Lifshitz, Course of Theoretical Physics III: Quantum Mechanics, Butterworth-Heinemann (1981) 689 p.

[17] H. B. Meyer, Lattice QCD and the Timelike Pion Form Factor, Phys.Rev.Lett. 107 (2011) 072002, arXiv:1105.1892.

[18] X.-d. Ji and C.-w. Jung, Studying hadronic structure of the photon in lattice QCD, Phys.Rev.Lett. 86 (2001) 208, hep-lat/0101014.

[19] S. Weinberg, The quantum theory of fields. Vol. 2: Modern applications, . Cambridge, UK: Univ. Pr. (1996) 489 p.

[20] S. L. Adler and W. A. Bardeen, Absence of higher order corrections in the anomalous axial vector divergence equation, Phys.Rev. 182 (1969) 1517-1536.

[21] C. Kim, C. Sachrajda, and S. R. Sharpe, Finite-volume effects for two-hadron states in moving frames, Nucl.Phys. B727 (2005) 218-243, hep-lat/0507006.

[22] M. T. Hansen and S. R. Sharpe, Multiple-channel generalization of Lellouch-Lüscher formula, Phys.Rev. D86 (2012) 016007, arXiv:1204.0826.

[23] J. Gasser and H. Leutwyler, $\eta \rightarrow 3 \pi$ to One Loop, Nucl.Phys. B250 (1985) 539.

[24] K. Polejaeva and A. Rusetsky, Three particles in a finite volume, Eur.Phys.J. A48 (2012) 67, arXiv:1203.1241.

[25] R. A. Briceno and Z. Davoudi, Three-particle scattering amplitudes from a finite volume formalism, arXiv:1212.3398 